\title{
An Unusual Case of Stroke and Fevers in a Traveler Returning from Arizona
}

\author{
Mikael Hanninen, Naeem Dean
}

Can. J. Neurol. Sci. 2008; 35: 119-121

Primary coccidioidal infection caused by inhalation of spores growing several inches below the surface of desert soil in the San Joaquim valley in southern Arizona and central California is well documented in the literature. Most infections resolve quickly with minimal symptoms, ${ }^{1}$ although some individuals develop a subacute process called "Valley Fever", which is characterized by shortness of breath, chest pain, cough and fevers lasting from weeks to months. Skin manifestations, including erythema nodosum and erythema multiforme may also be present and generally resolve with resolution of the respiratory tract illness. A recent retrospective study found that 6003 adult and 332 pediatric patients were hospitalized with endemic mycosis in $2002,{ }^{2}$ but the overall incidence has been estimated at about 150,000 infections per year. We report a unusual case of recurrent stroke temporally associated with a CNS Coccidiomycosis infection in a traveler to a region where the fungus is endemic.

\section{Case Presentation}

Mrs. S.M. was a previously healthy 74-year-old female who presented to an Edmonton hospital with multiple falls over the past three days. Her latest fall occurred at 0330 on June 14, 2006 as she was walking to the washroom. There was no significant trauma or loss of consciousness with these falls. At presentation in the Emergency Department, she also related a one day history of left-sided weakness commencing in the lower extremity and progressing to the left arm and face. Mrs. S.M. admitted to a past medical history of hypertension for which she took hydrochlorothiazide, but she denied any history of prior stroke, coronary or peripheral artery disease, diabetes, heart failure, dyslipidemia or arrhythmia. Physical examination revealed an elevated blood pressure of 162/79 and a left facial droop. There was mild weakness and reduced light touch sensation in the left arm and leg. Initial blood work, including Complete Blood Count, Electrolytes, Urea, Creatinine, Glucose and Troponin I was normal and Chest X-ray, and ECG showed no abnormalities. Although an initial CT of the head revealed only generalized atrophy, a clinical diagnosis of right lacunar stroke was made. The patient was admitted to the general medicine ward and started on daily anti-platelet therapy. The diagnosis of lacunar stroke was later confirmed with a gadolinium-enhanced MRI scan performed seven days after admission (Figure 1). No meningeal enhancement or other abnormalities were noted.

During her first day in hospital, a temperature of $38.5^{\circ} \mathrm{C}$ was noted and multiple blood and urine cultures were drawn. Over the following 12 days, Mrs. S. M. was treated for a positive urine culture $\left(10^{7}\right.$ cells $/ \mathrm{mL}$ of E. coli), a confluent opacity of her left lung based on chest X-ray as well as for Vulvovaginal Candidiasis with several courses of antibiotics and topical antifungals, but her intermittent fevers persisted. Over this time, her weakness and facial droop improved, but her fever endured and she started complaining of headaches (on direct questioning she admitted having intermittent headaches a couple of weeks prior to admission to the hospital). There were no new physical signs and repeated blood work (including blood cultures) was negative. On the morning of the eighth day of her admission, Mrs. S.M. became acutely confused. Blood, urine and vaginal cultures were repeated, but did not reveal any abnormalities. Protein electrophoresis and immuno-Electrophoresis were also normal. A lumbar puncture was performed and revealed a CSF leukocytosis of $688 \times 10^{6}$ cells / L (68\% lymphocyte and 32\% monocytes/macrophage), an elevated CSF protein of $2.02 \mathrm{~g} / \mathrm{L}$ (normal $0.15-0.45 \mathrm{~g} / \mathrm{L}$ ) and a CSF glucose of $1.6 \mathrm{mmol} / \mathrm{L}$ (normal range 2.2-4.4). Initial CSF testing for Herpes simplex Virus, West Nile Virus, and Acid Fast Bacilli were negative. Gram stain for bacteria and potassium hydroxide (KOH) prep for fungus were negative. In the mean time, the patient's fevers and confusion persisted. Computed tomogram of the chest, abdomen and pelvis revealed an area of scarring with multiple nodules measuring $4 \times 4.5 \times 1.5 \mathrm{~cm}$ at the left lower lung base.

Additional history was obtained from the patient's husband, revealing that the couple had recently returned from a yearly trip to Arizona. The husband also recalled driving across desert terrain in the San Joaquim valley in the last month or two. In the light of these findings, disseminated Coccidiomycosis was suspected. Serum and CSF IgM and IgG antibody testing for Coccidiomycosis was requested on the advice of Coccidiomycosis specialists at the Valley Fever Center for Excellence in Tuscon, Arizona and the diagnosis of coccidioidal meningitis was confirmed.

Mrs. S.M. was immediately started on oral fluconazole at a dose of $400 \mathrm{mg}$ daily. Several days after starting the fluconazole,

From the Royal Alexandra Hospital, Edmonton, Alberta, Canada.

Received April 5, 2007. Final Revisions Submitted August 11, 2007.

Reprint requests to: Mikael Hanninen, \#46 3075 Trelle Cres, Edmonton, Alberta T6R 3V5, Canada. 


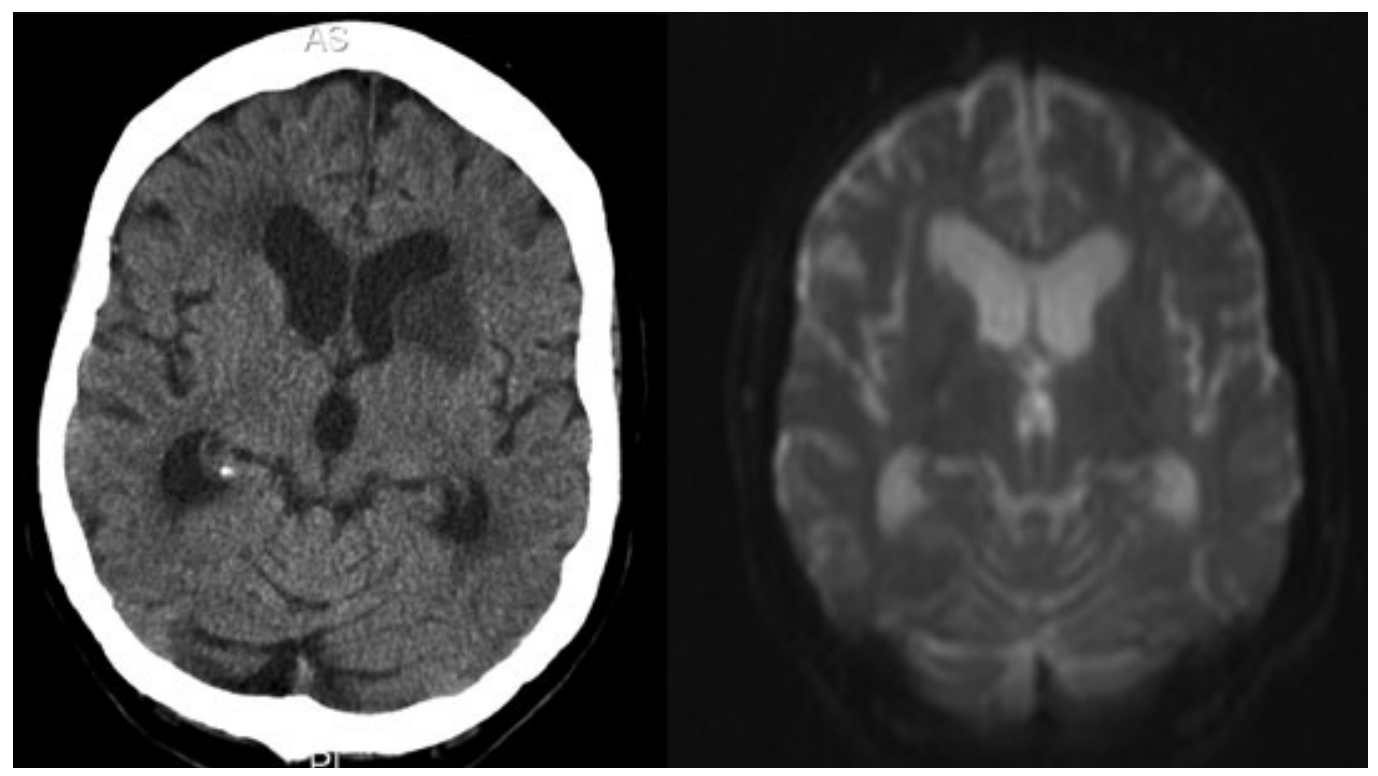

Figure 1. FLAIR acquisitions (left) and diffusion-weighted MR images (right) demonstrating a lacunar infarct of the right internal capsule.

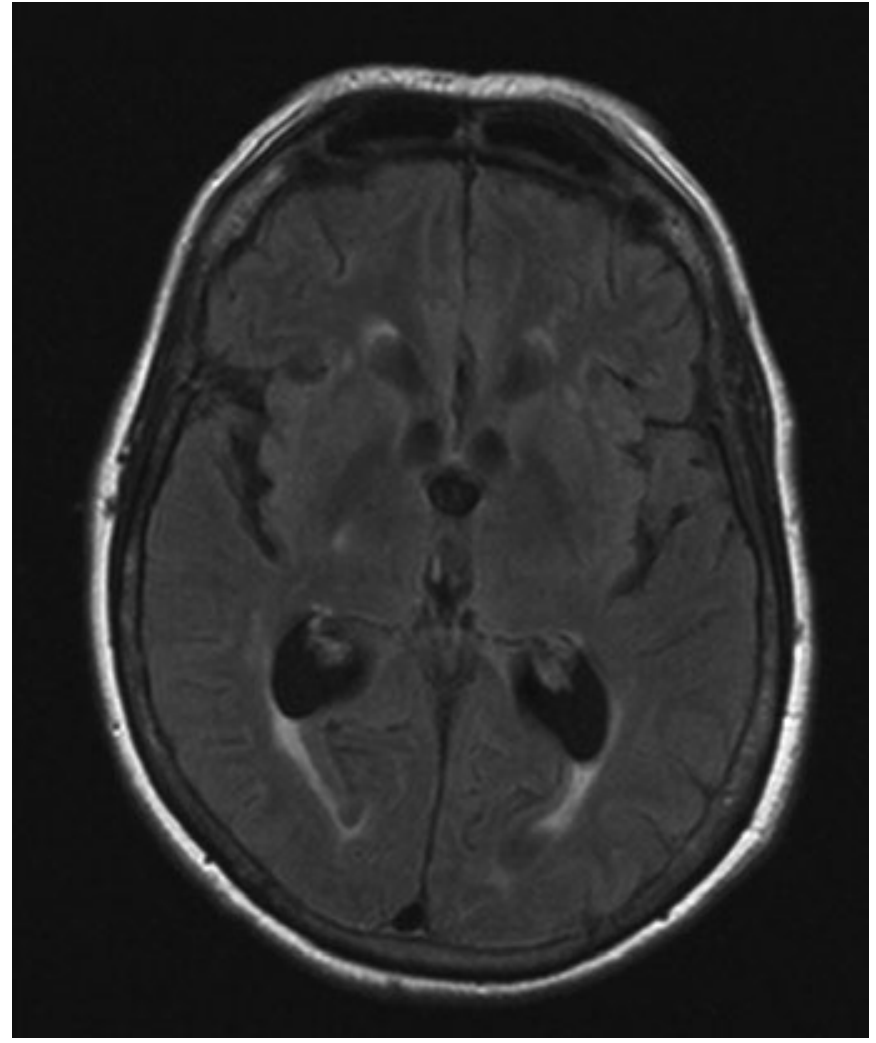

Figure 2. Non-enhanced CT of the head, showing an area of hypoattenuation involving the left caudate nucleus, anterior limb of the internal capsule and anterior lentiform nucleus, suggesting an ischemic infarct. she suffered an acute worsening of her headache and neurological status, along with new onset aphasia. The CT head revealed a new left-sided subcortical infarct (Figure 2). At this time, a 24-hour holter and trans-esophageal echocardiogram were performed to rule out a cardioembolic cause of her recurrent strokes.

Although she required gastrostomy tube placement to support her nutrional status, her confusion eventually improved and her intermittent fevers subsided. This clinical improvement was mirrored by an objective improvement in her CSF lymphocytosis (Figure 3). Many months after Mrs. S.M.'s initial presentation, her confusion and energy level improved to the point that she could participate in a hospital-based stroke rehabilitation program. A slow, but gradual recovery of her aphasia and mobility was eventually attained, but at the time of her discharge in January 2007 (nearly seven months after her initial presentation), she continued to have residual functional and cognitive impairments. After a long discussion with her family, it was decided that Mrs. S.M would be discharged to a long-term care facility. She was advised to continue suppressive oral fluconazole therapy indefinitely.

\section{DISCUSSION}

Coccidioidal meningitis is a rare and feared complication of infection with the fungi Coccidiodes immitis and Coccidiodes posadasil. Patients typically present with persistent headache, fever and mental status changes several months following primary exposure to Coccidiodes spp. Neurological deficits may also be present in severe cases. The CT scanning and MR imaging detects abnormalities such as hydrocephalus, basilar meningitis and cerebral infarction in $41.6 \%$ and $76 \%$ of patients 


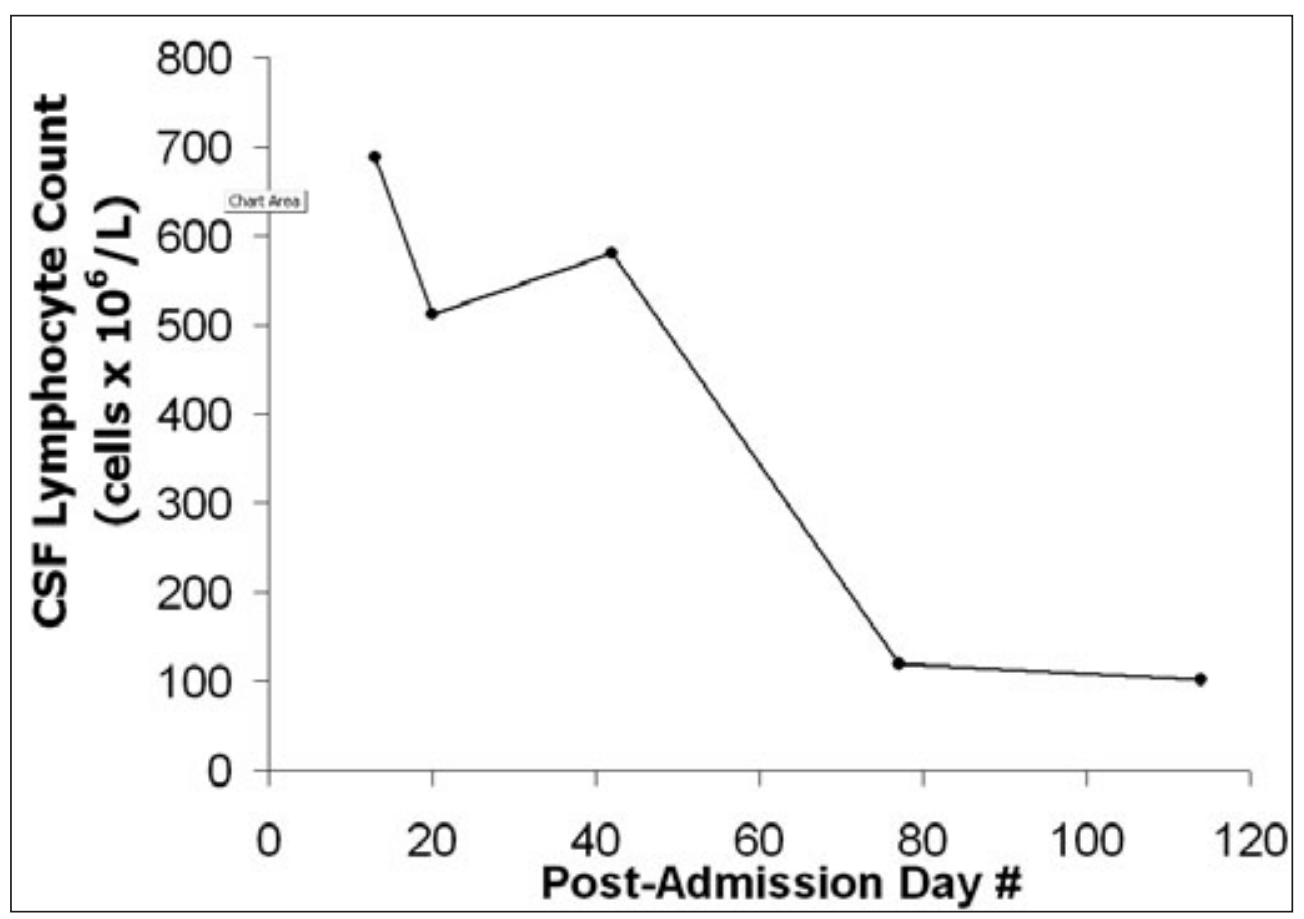

Figure 3. Progression of CSF lymphocytosis in Mrs S.M., a 74 year old woman with Coccidiomycosis meningitis who was treated with high dose fluconazole therapy

respectively. Elevated mortality rates are associated with hydrocephalus coexisting with infarction. ${ }^{3}$ Cerebral vasculitis with infarction has been reported to occur in conjuction with coccidioidal meningitis. ${ }^{4}$

The disease is nearly universally fatal without treatment, as evidenced by data from the pre-amphotericin B era. ${ }^{5}$ Oral fluconazole therapy has a response rate of approximately $79 \%$, with most improvement occurring four to eight months after starting treatment. Our case is particularly unusual in that the presenting complaints were all neurological in nature, with signs of infection occurring after admission to hospital. Despite a good biochemical and clinical response to anti-fungal therapy (improvement of CSF lymphocytosis, resolution of fevers and confusion), our patient's ultimate clinical outcome was similar to that of a patient suffering from bilateral ischemic strokes.

In 2004, over 430000 airplane passengers traveled between Canada and the state of Arizona. ${ }^{6}$ Arizona remains a particularly popular destination for Canadian "snowbirds", retirees who, while maintaining residential ties with Canada, spend their winters in the United States. In light of the large number of Canadians who visit Arizona and central California each year, it is important for Canadian physicians to remember to ask about travel history when dealing with atypical infectious presentations and fevers of unknown origin. An awareness of the signs and symptoms of infectious diseases endemic to different areas in North America is valuable in our world of increasingly frequent air travel.

\section{REFERENCES}

1. Smith, CE, Beard RR, Whiting EG, Rosenberg HG. Varieties of coccidioidal infection in relation to the epidemiology and control of the disease. Am J Public Health. 1946; 36:1394-402.

2. Chu JH, Feudtner C, Heydon K, Walsh TJ, Zaoutis TE. Hospitalizations for endemic mycoses: a population-based national study. Clin Infect Dis. 2006; 42(6):822-5.

3. Arsura EL, Johnson R, Penrose J, Stewart K, Kilgore W, Reddy $\mathrm{CM}$, et al. Neuroimaging as a guide to predict outcomes for patients with coccidioidal meningitis. Clin Infect Dis. 2005; 40(4):624-7.

4. Einstein HE, Holeman CW, Sandidge LL, Holden DH. Coccidioidal meningitis: the use of amphotericin B in treatment. Calif Med. 1961; 94:339-43.

5. Gottfredsson M, Perfect JR. Fungal meningitis. Semin Neurol. 2000; 20(3):307-22.

6. Statistics Canada. Air Passenger Origin and Destination, CanadaUnited States Report. 2004; Catalogue no. 51-205. 